\title{
BOUNDARY LAYER OF HYDROGEN CONCENTRATION UNDER PLASTIC DEFORMATION
}

\author{
A. K. Belyaev ${ }^{1}$, D. E. Mansyrev², V. A. Polyanskiy ${ }^{1 *}$, A. M. Polyanskiy ${ }^{3}$, \\ D. A. Tretyakov ${ }^{2}$, Yu. A. Yakovlev ${ }^{1}$ \\ ${ }^{1}$ Institute for Problems in Mechanical Engineering of the Russian Academy of Sciences, \\ 61, Bolshoy pr. V.O., St. Petersburg, Russian Federation \\ ${ }^{2}$ Peter the Great Saint-Petersburg Polytechnic University, \\ 29, Polytechnicheskaya, St. Petersburg, Russian Federation \\ ${ }^{3}$ Research \& Development Company Electronic \& Beam Technologies Ltd., \\ PO box 162, St. Petersburg, Russian Federation

\begin{abstract}
*Corresponding author. E-mail: vice.ipme@gmail.com
Address for correspondence: 61, Bolshoy pr. V.O., St. Petersburg, 199178, Russian Federation Tel.: +7 (812) 32147 78; fax: +7 (812) 3214771
\end{abstract}

\begin{abstract}
A new model of instability of uniform plastic deformation with the formation of Lüders bands on the surface of a material being deformed is experimentally confirmed. It has been found that nonuniform plastic deformations correlate with hydrogen concentration during the testing of metal specimens in the atmosphere at room temperature. The presence of additional pores and microcracks formed as a result of plastic deformation is the cause of the correlation between plastic deformations and hydrogen concentrations in aluminum alloys. The effect of the boundary layer in the distribution of hydrogen concentrations has been detected. It reflects the fact that all changes associated with mechanical and thermo-mechanical loading are localized in a thin boundary layer at the surface of a metal specimen. The wave approach to the analysis of the continuum equations and the model of the boundary layer of a bicontinuous medium containing hydrogen are used to describe the observed phenomena. The constructed model makes it possible to describe the development of plastic deformation as a wave process in the boundary layer of a bicontinuous medium.
\end{abstract}

Keywords: plastic deformation, Lüders bands, dissolved hydrogen, boundary-layer model, wave approach.

DOI: $10.17804 / 2410-9908.2017 .4 .032-043$

\section{References}

1. Von Gerstner F.A. Ueber die Festigkeit der Körper. Annalen der Physik, 1832, vol. 102, iss. 10, pp. 269-279. DOI: 10.1002/andp.18321021005.

2. Savart F. Recherches sur les vibrations longitudinales. Ann. Chim. Phys, 1837, vol. 65, pp. 337-402.

3. Portevin A., Le Chatelier F. Sur unphenomene observe lorsdel'essai de traction d'alliagesencours de transformation. Compt. Rend. Acad. Sci., Paris, 1923, vol. 176, pp. 507-510.

4. Penning P. Mathematics of the Portevin-Le Chatelier effect. Acta Metallurgica, 1972, vol. 20, no. 10, pp. 1169-1175. DOI: 10.1016/0001-6160(72)90165-4.

5. Lebyodkin M., Brechetz Y., Estrin Y., Kubin L. Statistical behaviour and strain localization patterns in the Portevin-Le Chatelier effect. Acta Materialia, 1996, vol. 44, no. 11, pp. 4531-4541. DOI: 10.1016/1359-6454(96)00076-6.

6. McCormick P.G. A model for the Portevin-Le Chatelier effect in substitutional alloys. Acta Metallurgica, 1972, vol. 20, no. 3, pp. 351-354. DOI: 10.1016/0001-6160(72)90028-4.

7. Kalk A., Nortmann A., Schwink C. Dynamic strain ageing and the boundaries of stable plastic deformation in Cu-Mn single crystals. Philosophical Magazine A, 1995, vol. 72, no. 5, pp. 1239-1259. DOI: 10.1080/01418619508236253. 
8. Leoni F., Zapperi S. Dislocation mutual interactions mediated by mobile impurities and the conditions for plastic instabilities. Physical Review E, 2014, vol. 89, no. 2, pp. 022403. DOI: 10.1103/PhysRevE.89.022403.

9. Kudinova N.R., Polyanskiy V.A., Polyanskiy A.M., Yakovlev Yu.A. Contribution of Surface Tension Energy during Plastic Deformation of Nanomaterials. Doklady Physics, 2016, vol. 61, no. 10, pp. 514-516. DOI: 10.1134/S1028335816100104.

10. Phillips A., Brick R.M. Effect of Quenching Strains on Lattice Parameter and Hardness Values of High Purity Aluminum-copper Alloys. Metals Technol., Tech. Paper., 1934, vol. 563, pp. 94-112.

11. Grishchenko A.I., Modestov V.S., Polyanskiy V.A., Tretyakov D.A., Shtukin L.V. Experimental investigation of the acoustic anisotropy field in the sample with a stress concentrator. St. Petersburg Polytechnical University Journal: Physics and Mathematics, 2017, vol. 3, iss. 1, pp. 77-82. DOI: 10.1016/j.spjpm.2017.02.005.

12. Belyaev A.K., Polyanskiy V.A., Lobachev A.M., Modestov V.S., Semenov A.S., Grishchenko A.I., Yakovlev Y.A., Shtukin L.V., Tretyakov D.A. Propagation of sound waves in stressed elasto-plastic material. In: Days on Diffraction 2016: Proceedings of the International Conference, St. Petersburg, 2016, pp. 56-61. DOI: 10.1109/DD.2016.7756813.

13. Belyaev A.K., Blekhman I.I., Polyanskiy V.A. Equation for the evolution of trapped hydrogen in an elastic rod subjected to high-frequency harmonic excitation. Acta Mech., 2016, vol. 227, pp. 1515-1518. DOI: 10.1007/s00707-015-1505-1.

14. Belyaev A.K., Polyanskiy V.A., Grishchenko A.I., Lobachev A.M., Mansyrev D.I., Modestov V.S., Pivkov A.V., Semenov A.S., Shtukin L.V., Tretyakov D.A., Yakovlev Yu.A. Application of the acoustic anisotropy approach for technical diagnostics of structures with large plastic deformations. In: AIP Conference Proceedings, AIP Publishing, 2016, vol. 1785, no. 1, pp. 030004. DOI: 10.1063/1.4967025.

15. Polyanskiy V.A., Belyaev A.K., Arseniev D.G., Yakovlev Yu.A., Polyanskiy A.M., Stoschka M. Measurement of dissolved hydrogen distributions after ultrasonic peening of heataffected zone of welded joint. AIP Conference Proceedings, AIP Publishing, 2016, vol. 1785, no. 1, pp. 030022-1-030022-4. DOI: 10.1063/1.4967043.

16. Belyaev A.K., Polyanskiy A.M., Polyanskiy V.A., Sommitsch Ch., Yakovlev Yu.A. Multichannel diffusion vs TDS model on example of energy spectra of bound hydrogen in 34CrNiMo6 steel after a typical heat treatment. International Journal of Hydrogen Energy, 2016, vol. 41, iss. 20, pp. 8627-8634. DOI: 10.1016/j.ijhydene.2016.03.198.

17. Belyaev A.K., Lobachev A.M., Modestov V.S., Pivkov A.V., Polyanskii V.A., Semenov A.S., Tret'yakov D.A., Shtukin L.V. Estimating the plastic strain with the use of acoustic anisotropy. Mechanics of Solids, 2016, vol. 51, iss. 5, pp. 606-611. DOI: $10.3103 / \mathrm{S} 0025654416050149$.

18. Hirao M., Pao Y.H. Dependence of acoustoelastic birefringence on plastic strains in a beam. The Journal of the Acoustical Society of America, 1985, vol. 77, no. 5, pp. 1659-1664. DOI: $10.1121 / 1.391964$.

19. Pao Y.H. Theory of acoustoelasticity and acoustoplasticity. In: Solid mechanics research for quantitative non-destructive evaluation, Springer, Netherlands, 1987, pp. 257-273.

20. Pao Y.H., Wu T.T., Gamer U. Acoustoelastic Birefringences in Plastically Deformed Solids: Part I. Theory. Journal of Applied Mechanics, 1991, vol. 58, no. 1, pp. 11-17. DOI: $10.1115 / 1.2897137$.

21. Alekseeva E.L., Alhimenko A.A., Belyaev A.K., Lobachev A.M., Polyanskiy V.A., Rostovykh G.N., Tretyakov D.A., Shtukin L.V., Yakovlev Y.A. Evaluation of stress-strain state and cracking of weatherproof structural steel by acoustoelasticity. Construction of Unique Buildings and Structures, 2016, vol. 12, pp. 33-44. DOI: 10.18720/CUBS.51.3. (In Russian).

22. NACE Standard TM0284-2003. Test Method. Evaluation of Pipeline and Pressure Vessel Steels for Resistance to Hydrogen-Induced Cracking. 
23. Andronov D.Yu., Arseniev D.G., Polyanskiy A.M., Polyanskiy V.A., Yakovlev Yu.A. Application of multichannel diffusion model to analysis of hydrogen measurements in solid. International. Journal of Hydrogen Energy, 2017, vol. 42, iss. 1, pp. 699-710. DOI: $10.1016 /$ j.ijhydene.2016.10.126.

24. Polyanskiy A.M., Polyanskiy V.A., Yakovlev Yu.A. Experimental determination of parameters of multichannel hydrogen diffusion in solid probe. International Journal of Hydrogen Energy, 2014, vol. 39, iss. 30, pp. 17381-17390. DOI: 10.1016/j.ijhydene.2014.07.080.

25. GOST 21132.1-98. Alyuminiy $i$ splavy alyuminievyye. Metod opredeleniya vodoroda $v$ tverdom metalle vakuum-nagrevom [Aluminum and Aluminum Alloys. Methods for the Determination of Hydrogen in Solid Metal by Vacuum Heating]. (In Russian).

26. Indeitsev D., Semenov B. About a model of structural-phase transformations under hydrogen influence. Acta Mechanica, 2008, vol. 195, pp. 295304. DOI: 10.1007/s00707-007-0568-z.

27. Belyaev A.K., Kudinova N.R., Polyanskiy V.A., Yakovlev Yu.A. The description of deformation and destruction of materials containing hydrogen by means of rheological model. St. Petersburg Polytechnical University Journal: Physics and Mathematics, 2015, vol. 3, pp. 57-65. DOI: 10.5862/JPM.225.14.

28. Gorsky W.S. Theorie des elastichen Nachwirkung in ungeordneten Mischkristallen (elastische Narchwirkung zweiter Art). Phys. Zeitschrift der Sowjetunion, 1935, vol. 8, pp. 457-471. 
Подана в журнал: 25.07 .2017

УДК 621.73.012

DOI: $10.17804 / 2410-9908.2017 .4 .032-043$

\title{
ПОГРАНИЧНЫЙ СЛОЙ КОНЦЕНТРАЦИИ ВОДОРОДА ПРИ ПЛАСТИЧЕСКОЙ ДЕФОРМАЦИИ
}

\author{
А. К. Беляев ${ }^{1}$, Д. Э. Мансырев ${ }^{2}$, В. А. Полянский ${ }^{1}$, А. М. Полянский ${ }^{3}$, \\ Д. А. Третьяков ${ }^{2}$ Ю. А. Яковлев ${ }^{1}$ \\ ${ }^{1}$ Институт Проблем Машиноведения Российской Академии Наук, \\ Васильевский остров, Большой проспект, 61, Санкт Петербург, Российская Федерация \\ ${ }^{2}$ Санкт-Петербургский политехнический университет Петра Великого, \\ Политехническая, 29, Санкт-Петербург, Российская Федерачия \\ ${ }^{3} О О О$ «Научно-производственный комплекс Электронные и Пучковые Технологии», \\ а/я 162, Санкт-Петербург, Российская Федерация \\ *Ответственный автор. Электронная почта: vice.ipme@gmail.com \\ Адрес для переписки: Васильевский остров, Большой проспект, 61, Санкт Петербург, Россия, 199178 \\ Тел.: +7 (812) 321-47-78; факс: +7 (812) 321-47-78
}

В статье проведено экспериментальное обоснование новой модели потери устойчивости равномерной пластической деформации, приводящей к появлению полос Людерса на поверхности деформируемого металла. Установлено, что при испытаниях металлических образцов в атмосфере при комнатной температуре неравномерные пластические деформации коррелированны с концентрацией водорода. В алюминиевых сплавах такая корреляция означает наличие дополнительных пор и микротрещин, возникших в результате пластической деформации. Обнаружен эффект пограничного слоя в распределении концентраций водорода, когда все изменения, связанные с механическим и термомеханическим нагружением, локализованы в тонком пограничном слое у поверхности металлической детали. Для описания обнаруженных явлений использована модель пограничного слоя двухконтинуальной сплошной среды, содержащей водород и волновой подход к анализу уравнений сплошной среды. Построенная модель позволяет описать развитие пластической деформации, как волновой процесс в пограничном слое двухконтинуальной сплошной среды.

Ключевые слова: пластическая деформачия, полосы Людерса, растворенный водород, модель пограничного слоя, волновой подход.

\section{1. Введение}

Большинство конструкционных металлов перед разрушением испытывает пластические деформации. Они наблюдаются даже при многоцикловой усталости. Поэтому процесс пластического течения является критически важным для прочности материалов и устойчивости конструкций из них.

Важной особенностью пластических деформаций реальных деталей, например, изготовленных из металлического проката, является большая неоднородность поля деформаций. Впервые этот эффект был замечен еще в 19-м в. (см. [1, 2]). Позже этот эффект получил название Портевена-Ле Шателье [3]. Связанный с ним механизм образования полос локализации пластической деформации (полос Людерса) является важным аспектом пластической деформации.

Предлагаются разные объяснения механизма такой локализации. Очевидно, что неравномерная деформация однородного материала под действием равномерной нагрузки является проявлением неустойчивости. Эта неустойчивость в любом случае имеет динамическую природу. 
Основная часть авторов считает, что причиной неустойчивости является наличие немонотонности на кривой напряжения-деформации $[4,5]$.

Другие авторы моделируют эффект локализации пластической деформации с помощью нелинейностей $[6,7]$ или путем введения в уравнения сплошной среды случайных величин [8]. Источником нелинейных зависимостей считается диффузия вакансий и связанных с ними дислокаций, а также нелинейные определяющие уравнения материала. Источником случайности считается процесс образования дислокаций.

Главной особенностью этих подходов является их фактическая одномерность. Все уравнения пишутся либо для однородного материала, либо для композита, состоящего из вкраплений одного материала в матрицу другого. Между тем установлено, что при пластическом деформировании поликристаллических материалов большую роль играют силы поверхностного натяжения или поверхностные силы кристаллитов [9]. Таким образом, необходимо учитывать наличие поверхностного слоя с особыми свойствами. Экспериментально большое влияние поверхностного слоя при пластической деформации было впервые замечено в начале 20-го в. [10].

Возникают вопросы: чем деформация поверхностного слоя отличается от деформации остального материала, каковы основные физические механизмы, как эти свойства моделировать. Настоящая статья предлагает ответы на поставленные вопросы.

\section{2. Методы исследования и экспериментальные результаты}

В качестве методов исследования мы использовали механические испытания на разрывных машинах INSTRON 8850, измерение параметров акустоупругости с помощью промышленного прибора ИН-5101А, измерение распределения концентраций растворенного водорода с помощью промышленного анализатора водорода AB-1, испытания на водородное растрескивание. Все исследовательское оборудование сертифицировано.

Были изучены образцы с большими пластическими деформациями [11-17]. Нагружение образцов проводилось в разрывных машинах с определением нагрузки и средних деформаций. Также производилась различная обработка образцов (пин-обработка, термомеханические нагрузки, шлифовка и т. д.).

Отмечено в литературе [18-20] и подтверждено нашими испытаниями [17], что процесс пластической деформации сопровождается резким изменением акустоупругости материала образцов. Принято считать, что это следствие разворота зерен металла и остаточных напряжений [19-20].

Численное конечно-элементное моделирование поля пластических деформаций показало, что наблюдается качественное несоответствие между примерно одинаковыми по величине пластическими деформациями в разных частях образцов и результатами измерения акустической анизотропии в этих областях [21]. Особенно сильно деформации и величина акустической анизотропии различались в зоне зарождения магистральной трещины, которая привела к разрушению образца. Этот факт послужил основанием для гипотезы о том, что микрорастрескивание может сильно влиять на величину акустической анизотропии.

Мы применили стандартный тест на водородное растрескивание для генерации водородных микротрещин в призматических $\left(15 \times 20 \times 100 \mathrm{mм}^{3}\right)$ образцах из стали 14 ХГНДЦ. Согласно стандарту [22], необходимо в течение 96 ч выдерживать образцы в деаэрированном водном растворе $5 \%$ по массе $\mathrm{NaCl}$ и $0,5 \%$ по массе $\mathrm{CH}_{3} \mathrm{COOH}$. В процессе проведения теста в растворе создается и поддерживается концентрация 1000 мг/л $\mathrm{H}_{2} \mathrm{~S}$ методом барботирования газообразным сероводородом. После этого проводятся измерения параметров микротрещин на шлифах образцов.

Исследования образцов после стандартного теста на водородное растрескивание сталей, согласно стандарту [22], позволили подтвердить гипотезу о том, что микрорастрескивание вызывает существенное изменение величины акустической анизотропии [21].

Следовательно, микрорастрескивание поверхности может стать одним из ведущих механизмов изменения ее структуры при пластической деформации. 
Для проверки этой гипотезы мы использовали тот факт, что алюминиевые сплавы являются насыщенными растворами водорода. Это происходит из-за специфических свойств алюминиевой матрицы. В расплаве алюминия насыщенная концентрация водорода в несколько раз больше, чем в твердом состоянии, поэтому у твердого алюминия она почти всегда предельная. «Лишний» водород, который не успел улетучиться в атмосферу при кристаллизации, весь находится в порах и трещинах. Если концентрация водорода увеличивается при резании, пластическом деформировании или иной обработке твердого алюминиевого сплава, то с большой вероятностью это связано с образованием новых пор, микротрещин и других дефектов в объеме и на поверхности металла.

Мы провели измерения пространственного распределения водорода в образцах из алюминиевого сплава АМц, полученных в результате циклического и статического нагружения в разрывной машине. Образцы были вырезаны из плит толщиной 16 и 20 мм и имели стандартную гантелевидную форму (рис. 1). Штриховой линией показана схема нарезки призматических образцов для измерения распределения водорода. Характерный размер призматических образцов 6 мм.

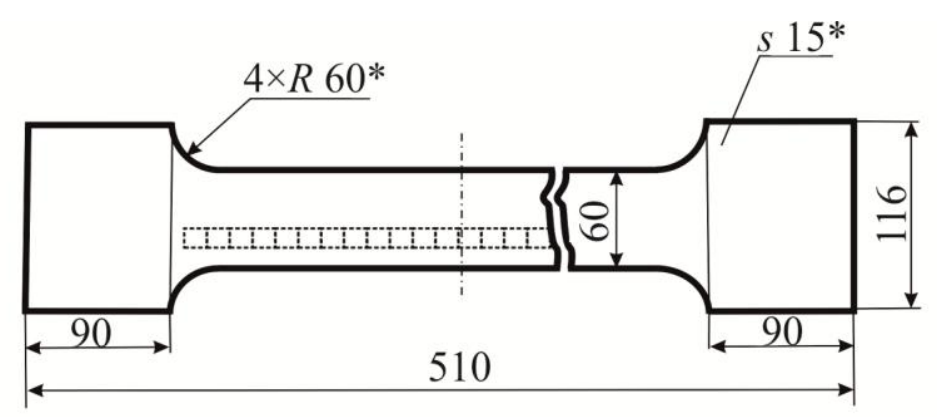

Рис. 1. Образцы после механических испытаний

Нарезка образцов для измерения концентрации водорода производилась ручной пилой, чтобы не допустить их нагревания и связанного с ним перераспределения водорода. С части образцов был удален слой, связанный с поверхностью исходного большого образца толщиной около 500 мкм. Результаты измерений представлены на рис. 2 в зависимости от удаления места разрыва образца в миллиметрах.

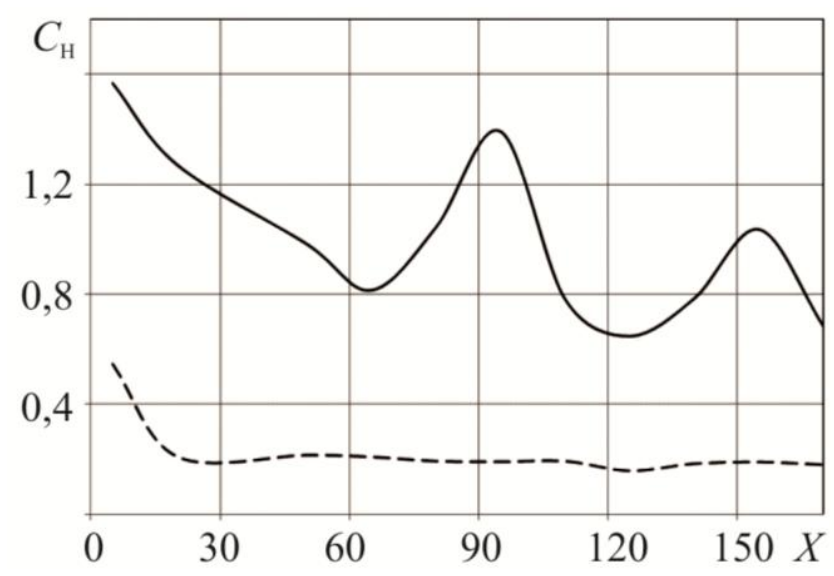

Рис. 2. Зависимость концентрация растворенного водорода в массовых долях $C_{\mathrm{H}}\left(\right.$ млн. $\left.^{-1}\right)$ от расстояния $x$ (в мм) от геометрического центра образцов до линии излома: сплошная линия - образцы с поверхностным слоем; пунктирная - с удаленным слоем поверхности исходного образца толщиной 500 мкм 
Методики измерений описаны в $[23,24]$. Определение растворенного водорода проводилось согласно стандарту [25]. Наиболее близкие к месту разрушения образцы содержали часть поверхности разрушения.

Хорошо видно на рис. 2, что все поры и микротрещины, образовавшиеся в результате деформации, сосредоточены в тонком поверхностном слое металла. Установленная нами новая закономерность позволяет рассматривать материал как трехмерную среду, содержащую тонкий поверхностный слой с особыми свойствами. Этот поверхностный слой содержит значительные количества водорода, что позволяет использовать для его моделирования концепцию двухконтинуальной сплошной среды, предложенной в $[26,27]$.

\section{3. Теоретическая модель}

Основой модели являются представления о наличии внутри металла водорода с различными энергиями связи. Водород с низкой энергией связи является диффузноподвижным и стремится к зонам растягивающих напряжений (эффект Горского [28]). Накопление водорода в зоне разрушения происходит как в результате сорбции из окружающей среды, так и при перераспределении его естественных концентраций внутри материала. Водород с низкой энергией связи имеет слабое взаимодействие с металлом. Водород с высокой энергией связи, напротив, взаимодействует с металлом очень интенсивно, но при этом очень медленно перемещается внутри металла. Механические свойства вмещающего материала ухудшаются, прежде всего, вследствие жесткого взаимодействия, так как водород, присоединившийся к атомам металла, отнимает энергию у кристаллической структуры.

Таким образом, процесс увеличения энергии связи водорода сопровождается уменьшением на ту же величину энергии связи внутри кристаллической структуры и связанной с этим деградацией ее механических характеристик [27]. Следовательно, материал, содержащий водород, можно представить состоящим из двух взаимодействующих континуумов. Масса диффузно-подвижного водорода внутри произвольного объема $V$ записывается в виде следующего интеграла:

$$
m_{H}^{-}=\int_{V} \rho_{H}^{-} d V
$$

Здесь $m_{\mathrm{H}}^{-}, \rho_{\mathrm{H}}^{-}$- масса и объемная плотность диффузно-подвижного водорода.

В дифференциальной форме уравнение баланса массы водорода имеет вид:

$$
\frac{\partial \rho_{\mathrm{H}}^{-}}{\partial t}+\nabla \cdot\left(\mathbf{v}_{\mathrm{H}}^{-} \rho_{\mathrm{H}}^{-}\right)=j_{\mathrm{H}}^{-},
$$

где $j_{\mathrm{H}}^{-}-$объемный источник диффузно-подвижного водорода; $\mathbf{v}_{\mathrm{H}}^{-}-$вектор скорости его перемещения.

Аналогичный баланс массы можно написать для связанного водорода:

$$
\frac{\partial n_{H}^{+}}{\partial t}+\nabla \cdot\left(\mathbf{v}_{H}^{+} n_{H}^{+}\right)=\frac{j_{H}^{+}}{m_{H}}
$$


Здесь $n_{H}^{+}$и $\mathbf{v}_{H}^{+}$- концентрация и скорость частиц связанного водорода, а потоки водорода $-j_{H}^{-}=j_{H}^{+}=j$ определяют его перераспределение внутри материала по энергиям связи. Из-за малости концентрации можно принять линеаризованную зависимость потока от концентрации водорода:

$$
j=\alpha n_{H}^{-}-\beta n_{H}^{+}
$$

Коэффициенты $\alpha$ и $\beta$, описывающие сорбцию и десорбцию связанного водорода, определяют перераспределение концентраций водорода по энергиям связи.

С точки зрения реологии вмещающий материал является континуумом, который моделирует твердое тело и присоединенные к его частицам водород. Уравнение динамики первого континуума имеет вид:

$$
\nabla \cdot \boldsymbol{\tau}=\left(\rho_{0}+\rho_{H}^{+}\right) \frac{\partial \mathbf{v}_{H}^{+}}{\partial t}+j \mathbf{v}_{H}^{+}+\mathbf{R}
$$

где $\boldsymbol{\tau}$ - тензор напряжений; $\mathbf{R}$ - сила взаимодействия вмещающей среды и диффузноподвижного водорода.

Второй континуум, моделирующий диффузно-подвижный водород, описывается уравнениями идеального газа. С учетом того, что давление газа $p$ имеет положительный знак при сжатии, динамическое уравнение имеет вид:

$$
-\nabla p=\rho_{H}^{-} \frac{\partial \mathbf{v}_{H}^{-}}{\partial t}-j \mathbf{v}_{H}^{-}-\mathbf{R}
$$

Модуль вектора скорости частиц диффузно-подвижного водорода $\mathbf{v}_{H}^{-}$имеет малую величину, так как эта скорость описывает диффузию водорода в твердом теле.

В тензоре напряжений $\tau$ можно выделить сферическую часть и девиатор

$$
\boldsymbol{\tau}=\sigma \mathbf{I}+\mathbf{s},
$$

где $\sigma$ - среднее напряжение; $\mathbf{s}$ - девиатор; $\mathbf{I}$ - единичный тензор.

Эффект Горского состоит в том, что энергия объемного растяжения определяет диффузию водорода и взаимодействие водорода с матрицей вмещающего материала. Следовательно, можно считать, что только сферическая часть тензора напряжений будет зависеть от второго континуума. Уравнения динамики двухконтинуальной сплошной среды в этом случае принимают вид:

$$
\begin{aligned}
& \nabla \sigma=\left(\rho_{0}+\rho_{H}^{+}\right) \frac{\partial \mathbf{v}_{H}^{+}}{\partial t}+j \mathbf{v}_{H}^{+}+\mathbf{R}, \\
& -\nabla p=\rho_{H}^{-} \frac{\partial \mathbf{v}_{H}^{-}}{\partial t}-j \mathbf{v}_{H}^{-}-\mathbf{R} .
\end{aligned}
$$


После линеаризации уравнений для тонкого поверхностного слоя получается уравнение динамики концентрации связанного водорода $n^{+}$при одноосной деформации образца вдоль оси $x$ :

$$
\frac{\partial^{2} n^{+}}{\partial t^{2}}+(\alpha+\beta) \frac{\partial n^{+}}{\partial t}-\frac{3 k T}{2 m_{H} F\left(\varepsilon_{s t}\right)}\left[\beta \frac{\partial^{2} n^{+}}{\partial x^{2}}+\frac{\partial^{3} n^{+}}{\partial t \partial x^{2}}\right]=0 .
$$

Здесь $k$ - постоянная Больцмана; $T$ - абсолютная температура подвижного континуума; $m_{H}-$ масса «частиц» водорода. Параметр $F\left(\varepsilon_{s t}\right)$, зависящий от величины статической деформации $\varepsilon_{s t}$, является мерой эффективной площади поперечного сечения каналов диффузии подвижного водорода, которая определяется свойствами материала (размером зерна, наличием пор и т. д.).

Мы предполагаем, что поверхностный слой соединен с основным материалом, который медленно растягивается по закону $\varepsilon_{s t}=\varepsilon_{s t}(t)$. Именно так выглядят натурные испытания образцов. Деформации являются малой величиной, а $F\left(\varepsilon_{s t}\right)=F_{0}-k_{0} \varepsilon_{s t}^{2}$ убывает с ростом деформаций, тогда в первом приближении уравнение (8) может быть записано в виде:

$$
\frac{\partial^{2} n^{+}}{\partial t^{2}}+(\alpha+\beta) \frac{\partial n^{+}}{\partial t}-\frac{3 k T}{2 m_{H} F_{0}}\left(1+\frac{k_{0}}{F_{0}} \varepsilon_{s t}^{2}\right)\left[\beta \frac{\partial^{2} n^{+}}{\partial x^{2}}+\frac{\partial^{3} n^{+}}{\partial t \partial x^{2}}\right]=0
$$

В этом уравнении можно выделить малый параметр, что позволяет применить асимптотические методы. Порождающее решение получается из следующего

$$
\frac{\partial^{2} n^{+}}{\partial t^{2}}+(\alpha+\beta) \frac{\partial n^{+}}{\partial t}-\frac{3 k T}{2 m_{H} F_{0}}\left[\beta \frac{\partial^{2} n^{+}}{\partial x^{2}}+\frac{\partial^{3} n^{+}}{\partial t \partial x^{2}}\right]=0 \text {. }
$$

Затем, вычитая порождающее решение из (9), можно получить следующее приближение, которое будет иметь порядок $O\left(\varepsilon_{s t}^{2}\right)$. Порождающее решение можно рассматривать как волновое, чему способствует осциллирующий характер распределения концентраций водорода, наблюдаемый экспериментально (рис. 2).

Будем разыскивать решение в виде $n^{+}=n^{+}(\theta), \theta=\kappa x-\lambda t+\varphi$, где $\kappa, \lambda$ - волновые числа, $\varphi$ - сдвиг фазы, тогда:

$$
\lambda^{2} \frac{d^{2} n^{+}}{d \theta^{2}}-(\alpha+\beta) \lambda \frac{d n^{+}}{d \theta}-\frac{3 k T}{2 m_{H} F_{0}}\left[\beta \kappa^{2} \frac{d^{2} n^{+}}{d \theta^{2}}-\kappa^{2} \lambda \frac{d^{3} n^{+}}{d \theta^{3}}\right]=0
$$

Откуда, обозначив $\gamma=\frac{3 k T}{2 m_{H} F_{0}}$, получим:

$$
\gamma \kappa^{2} \lambda \frac{d^{3} n^{+}}{d \theta^{3}}+\left(\lambda^{2}-\gamma \kappa^{2} \beta\right) \frac{d^{2} n^{+}}{d \theta^{2}}-(\alpha+\beta) \lambda \frac{d n^{+}}{d \theta}=0
$$


Собственные числа данного уравнения с постоянными коэффициентами определяются уравнением:

$$
\begin{gathered}
\gamma \kappa^{2} \lambda p^{3}+\left(\lambda^{2}-\gamma \kappa^{2} \beta\right) p^{2}-(\alpha+\beta) \lambda p=0 ; \\
p_{1}=0, p_{2,3}=\frac{-\left(\lambda^{2}-\gamma \kappa^{2} \beta\right) \pm \sqrt{D}}{2 \gamma \kappa^{2} \lambda}, D=\lambda^{4}+\gamma^{2} \kappa^{4} \beta^{2}+2 \lambda^{2} \gamma \kappa^{2} \beta+4 \lambda^{2} \gamma \kappa^{2} \alpha .
\end{gathered}
$$

Дисперсионное соотношение:

$$
-i \gamma k^{2} \omega-\omega^{2}+\not k^{2} \beta-i(\alpha+\beta) \omega=0,
$$

откуда

$$
k^{2}=\frac{\omega^{2}+i(\alpha+\beta) \omega}{\gamma \beta-i \gamma \omega}=\frac{1}{\gamma} \frac{-\alpha \omega^{2}+i\left((\alpha+\beta) \omega \beta+\omega^{3}\right)}{\beta^{2}+\omega^{2}} .
$$

Таким образом, затухание по времени сочетается с положительной вещественной частью экспоненты по координате и наоборот.

С другой стороны, в случае $\kappa=i k, \lambda=i \omega$, получим один нулевой и два чисто мнимых корня разного знака вида:

$$
p_{2,3}=\frac{\left(\omega^{2}-\gamma k^{2} \beta\right) \pm \sqrt{D}}{2 \gamma k^{2} \omega} i, D=\omega^{4}+\gamma^{2} k^{4} \beta^{2}+2 \omega^{2} \gamma k^{2} \beta+4 \omega^{2} \gamma k^{2} \alpha \text {. }
$$

Это позволяет записать решение в виде:

$$
n^{+}=n^{+}(\theta)=A_{1}+A_{2} \exp \left(p_{2} \theta\right)+A_{3} \exp \left(p_{3} \theta\right)
$$

где второе слагаемое будет давать волну, затухающую в пространстве, но нарастающую по времени экспоненциально до бесконечности, а третье - наоборот, затухающую во времени уединенную волну с нарастанием амплитуды по пространству.

Для удовлетворения произвольным граничным условиям нужны все три функции, так что нарастания амплитуды до бесконечности не избежать в линейной постановке. С другой стороны, есть устойчивое решение $n^{+}=n^{+}(\theta)=A_{1}$. Таким образом, если концентрация водорода равномерная, то она может сохраняться сколь угодно долго. Однако при любом перекосе в граничных условиях и неоднородности появляются все три решения, и амплитуда концентрации будет экспоненциально нарастать. Этим объясняется критическое влияние малых неоднородностей (ловушек водорода) и неравномерных граничных условий на устойчивость всей системы.

\section{4. Заключение}

Таким образом, порождающее решение уравнений концентрации водорода, связанного с матрицей материала, в тонком пограничном слое будет волновым для любого неоднородного распределения концентрации водорода по поверхности.

В этом решении всегда присутствует комплексная частота колебаний, которая означает наличие экспоненциально-нарастающей во времени амплитуды концентрационной волны, которая в силу уравнений двухконтинуальной сплошной среды приводит к деградации механических характеристик материала в зонах с максимальной концентрацией водорода. Такая 
неоднородность характеристик легко объясняет возникновение полос локализации пластической деформации, тем более что они часто имеют характерную длину волны, т. е. отстоят на приблизительно равном расстоянии друг от друга на поверхности образца.

Новый подход имеет большое практическое значение, так как позволяет связать концентрацию водорода с пластической деформацией материала. Таким образом, измерения концентрации растворенного водорода можно использовать для оценки остаточного ресурса материалов и конструкций из них.

\section{Благодарность}

Исследование выполнено при поддержке РФФИ, проекты No. 15-08-03112-а и 17-08-00783-a.

\section{Литература}

1. Von Gerstner F. A. Ueber die Festigkeit der Körper // Annalen der Physik. - 1832. Vol. 102, iss. 10. - P. 269-279. - DOI: 10.1002/andp.18321021005/.

2. Savart F. Recherches sur les vibrations longitudinales // Ann. Chim. Phys. - 1837. Vol. 65. - P. 337-402.

3. Portevin A., Le Chatelier F. Sur un phenomene observe lors del'essai de traction d'alliages en cours de transformation // Compt. Rend. Acad. Sci. - Paris. - 1923. - Vol. 176. - P. 507-510.

4. Penning P. Mathematics of the Portevin-Le Chatelier effect // Acta Metallurgica. - 1972. Vol. 20, no. 10. - P. 1169-1175. - DOI: 10.1016/0001-6160(72)90165-4.

5. Lebyodkin M., Brechetz Y., Estrin Y., Kubin L. Statistical behaviour and strain localization patterns in the Portevin-Le Chatelier effect // Acta materialia. - 1996. - Vol. 44, no. 11. - P. 45314541. - DOI: 10.1016/1359-6454(96)00076-6.

6. McCormick P. G. A model for the Portevin-Le Chatelier effect in substitutional alloys // Acta Metallurgica. - 1972. - Vol. 20, no. 3. - P. 351-354. - DOI: 10.1016/0001-6160(72)90028-4.

7. Kalk A., Nortmann A., Schwink C. Dynamic strain ageing and the boundaries of stable plastic deformation in $\mathrm{Cu}-\mathrm{Mn}$ single crystals // Philosophical Magazine A. - 1995. - Vol. 72, no. 5. P. 1239-1259. - DOI: 10.1080/01418619508236253.

8. Leoni F., Zapperi S. Dislocation mutual interactions mediated by mobile impurities and the conditions for plastic instabilities // Physical Review E. - 2014. - Vol. 89, no. 2. - P. 022403. DOI: 10.1103/PhysRevE.89.022403.

9. Contribution of Surface Tension Energy during Plastic Deformation of Nanomaterials / N. R. Kudinova, V. A. Polyanskiy, A. M. Polyanskiy, Yu. A. Yakovlev // Doklady Physics. - 2016. Vol. 61, no. 10. - P. 514-516. - DOI: 10.1134/S1028335816100104.

10. Phillips A., Brick R. M. Effect of Quenching Strains on Lattice Parameter and Hardness Values of High Purity Aluminum-copper Alloys // Metals Technol., Tech. Paper. - 1934. Vol. 563. P. 94-112.

11. Experimental investigation of the acoustic anisotropy field in the sample with a stress concentrator / A. I. Grishchenko, V. S. Modestov, V. A. Polyanskiy, D. A. Tretyakov, L. V. Shtukin // St. Petersburg Polytechnical University Journal: Physics and Mathematics. - 2017. - Vol. 3, iss. 1. P. 77-82. - DOI:10.1016/j.spjpm.2017.02.005.

12. Propagation of sound waves in stressed elasto-plastic material / A. K. Belyaev, V. A. Polyanskiy, A. M. Lobachev, V. S. Modestov, A. S. Semenov, A. I. Grishchenko, Y. A. Yakovlev, L. V. Shtukin, D. A. Tretyakov // Proceedings of the International Conference: Days on Diffraction 2016. - St. Petersburg, 2016. - P. 56-61. - DOI: 10.1109/DD.2016.7756813.

13. Belyaev A. K., Blekhman I. I., Polyanskiy V. A. Equation for the evolution of trapped hydrogen in an elastic rod subjected to high-frequency harmonic excitation// Acta Mech. - 2016. Vol. 227. - P. 1515-1518. - DOI: 10.1007/s00707-015-1505-1. 
14. Application of the acoustic anisotropy approach for technical diagnostics of structures with large plastic deformations / A. K. Belyaev, V. A. Polyanskiy, A. I. Grishchenko, A. M. Lobachev, D. I. Mansyrev, V. S. Modestov, A. V. Pivkov, A. S. Semenov, L. V. Shtukin, D. A. Tretyakov, Yu. A. Yakovlev // AIP Conference Proceedings. - AIP Publishing, 2016. - Vol. 1785, no. 1. P. 030004. - DOI:10.1063/1.4967025.

15. Measurement of dissolved hydrogen distributions after ultrasonic peening of heataffected zone of welded joint / V. A. Polyanskiy, A. K. Belyaev, D. G. Arseniev, Yu. A. Yakovlev, A. M. Polyanskiy, M. Stoschka // AIP Conference Proceedings. - AIP Publishing, 2016. Vol. 1785, no. 1. - P. 030022-1-030022-4. - DOI: 10.1063/1.4967043.

16. Multichannel diffusion vs TDS model on example of energy spectra of bound hydrogen in 34CrNiMo6 steel after a typical heat treatment / A. K. Belyaev, A. M. Polyanskiy, V. A. Polyanskiy, Ch. Sommitsch, Yu. A. Yakovlev // International Journal of Hydrogen Energy. 2016. - Vol. 41, iss. 20. - P. 8627-8634. - DOI: 10.1016/j.ijhydene.2016.03.198.

17. Estimating the plastic strain with the use of acoustic anisotropy / A. K. Belyaev, A. M. Lobachev, V. S. Modestov, A. V. Pivkov, V. A. Polyanskii, A. S. Semenov, D. A. Tret'yakov, L. V. Shtukin // Mechanics of Solids. - 2016. - Vol. 51, iss. 5. - P. 606-611. DOI: $10.3103 / \mathrm{S} 0025654416050149$.

18. Hirao M., Pao Y. H. Dependence of acoustoelastic birefringence on plastic strains in a beam // The Journal of the Acoustical Society of America. - 1985. - Vol. 77, no. 5. - P. 1659-1664. DOI: $10.1121 / 1.391964$.

19. Pao Y. H. Theory of acoustoelasticity and acoustoplasticity // Solid mechanics research for quantitative non-destructive evaluation. - Netherlands : Springer, 1987. - P. 257-273.

20. Pao Y. H., Wu T. T., Gamer U. Acoustoelastic Birefringences in Plastically Deformed Solids: Part I - Theory // Journal of Applied Mechanics. - 1991. - Vol. 58, no. 1. - P. 11-17. DOI: $10.1115 / 1.2897137$.

21. Evaluation of stress-strain state and cracking of weatherproof structural steel by acoustoelasticity / E. L. Alekseeva, A. A. Alhimenko, A. K. Belyaev, A. M. Lobachev, V. A. Polyanskiy, G. N. Rostovykh, D. A. Tretyakov, L. V. Shtukin, Y. A. Yakovlev // Construction of Unique Buildings and Structures. - 2016. - Vol. 12. - P. 33-44. DOI: 10.18720/CUBS.51.3.

22. NACE Standard TM0284-2003. Test Method. Evaluation of Pipeline and Pressure Vessel Steels for Resistance to Hydrogen-Induced Cracking.

23. Application of multichannel diffusion model to analysis of hydrogen measurements in solid / D. Yu. Andronov., D. G. Arseniev, A. M. Polyanskiy, V. A. Polyanskiy, Yu. A. Yakovlev // International Journal of Hydrogen Energy. - 2017. - Vol. 42, iss. 1. - P. 699-710. DOI: 10.1016/j.ijhydene.2016.10.126.

24. Polyanskiy A. M., Polyanskiy V. A., Yakovlev Yu. A. Experimental determination of parameters of multichannel hydrogen diffusion in solid probe// International Journal of Hydrogen Energy. - 2014. - Vol. 39, iss. 30. - P. 17381-17390. - DOI: 10.1016/j.ijhydene.2014.07.080.

25. GOST 21132.1-98. Aluminium and aluminium alloys. Methods for determination of hydrogen in solid metal by vacuum hot extraction.

26. Indeitsev D., Semenov B. About a model of structural-phase transformations under hydrogen influence // Acta Mechanica. - 2008. - Vol. 195. - P. 295-304. - DOI: 10.1007/s00707-007-0568-Z.

27. The description of deformation and destruction of materials containing hydrogen by means of rheological model / A. K. Belyaev, N. R. Kudinova, V. A. Polyanskiy, Yu. A. Yakovlev // St. Petersburg Polytechnical University Journal. Physics and Mathematics. - 2015. - Vol. 3. P. 57-65. - DOI: 10.5862/JPM.225.14.

28. Gorsky W. S. Theorie des elastichen Nachwirkung in ungeordneten Mischkristallen (elastische Narchwirkung zweiter Art) // Phys. Zeitschrift der Sowjetunion. - 1935. - Vol. 8. P. 457-471. 